\title{
Daily Physical Activity Improves Vascular Function and Motor Skills in Children
}

\author{
Sascha Ketelhut ${ }^{1,2}$, Kerstin Ketelhut ${ }^{3}$, Claudia Hacke ${ }^{4}$ and Reinhard Günter Ketelhut ${ }^{1,5,6}$ \\ 1. Charité-University Medicine Berlin, Berlin 10117, Germany \\ 2. Institute of Sports Science, Martin-Luther-University Halle-Wittenberg, Halle (Saale) 06120, Germany \\ 3. Medical School Berlin, Berlin 12247, Germany \\ 4. Institute of Medical Biometry and Epidemiology, University Medical Centre Hamburg-Eppendorf, Hamburg 20251, Germany \\ 5. Medical Center Berlin (MCB), Berlin 10559, Germany \\ 6. Institute of Sports Medicine, Humboldt-University Berlin, Berlin 10115, Germany
}

\begin{abstract}
The literature is consistent that regular physical activity in children has to be increased in order to compensate the negative health effects resulting from growing sedentary lifestyle. Thus, we analyzed in 45 students (aged $6.6 \pm 0.7$ years) the effects of a regular exercise intervention, on top of the normal physical education classes, on motor performance (MP) and hemodynamic parameters. The students were randomized either to an intervention (INT) $(n=23)$ or a control (CON) $(n=22)$ group. Throughout a 9 months period, INT received additional exercise interventions two days a week for 45 minutes. The main outcome measures were MP, peripheral and central blood pressure (BP), augmentation index (Alx) and aortic pulse wave velocity (aPWV). After the exercise intervention, we found significant $(P<0.05)$ positive effects on various test items of MP and significant decreases in peripheral and central systolic BP in INT. In contrast, in CON the respective parameters increased in CON after the observation period. Moreover, aPWV decreased significantly in INT $(P=0.047)$ and increased in CON, thus resulting in a significant difference in the mean change $(P$ $=0.043$ ). There were no considerable changes in AIx in both groups. In conclusion, we demonstrated that an increase in regular physical activity has a positive influence on development of MP and hemodynamic parameters even in early childhood. The findings of this study suggest that an increase in exercise time should be initiated as early as possible to improve motor ability and prevent future cardiovascular risk.
\end{abstract}

Key words: Physical fitness in children, exercise and blood pressure, arterial stiffness, motor performance.

\section{Introduction}

Today's children experience an increasing lack of physical activity and a rising evidence of cardiovascular risk factors such as overweight and elevated blood pressure (BP).

Hypertension is one of the leading causes of global disease burden [1] and a major independent risk factor for cardiovascular diseases. Even subjects with pre-hypertension are at increased risk for future cardiovascular morbidity and mortality [2]. The only recommended treatment for pre-hypertension and prevention of hypertension is to modify lifestyle,

Corresponding Author: Sascha Ketelhut, M.A., ISHRF, research fields: sports science, sports medicine, hemodynamics. typically by normalizing elevated body-weight and increasing physical activity [3].

Cardiovascular risks and diseases originate in the first decade of life [4]. Arterial hypertension appears to have its onset in childhood as well [5] and high BP at a young age predicts cardiovascular morbidity and mortality decades later [6]. An earlier study suggests that an alarming number of youth have hypertension or nearly meet the definition of hypertension with documented BP in the hypertensive range [7] and multiple studies have demonstrated tracking of hypertension into adulthood. Therefore, early diagnosis, evaluation, and treatment but most of all prevention of hypertension are important to avoid future organ damage and alleviate the health risks 
associated with future arterial hypertension.

Regular physical exercise promotes health and improves cardiovascular function not only in adults but in youth as well [8]. New research suggests that the level of cardio-respiratory fitness at an early age may predict long-term cardiac outcomes [9]. In a large cohort, low aerobic capacity was associated with increased risk of hypertension, independent of family history and socioeconomic factors [10].

Emerging evidence now suggests that in contrast to pressure measured in the brachial artery, central pressure is more strongly associated with preclinical organ damage such as left ventricular hypertrophy, carotid intima-media thickness and pulse wave velocity [11] and is better related to future cardiovascular events as well [12]. Antihypertensive therapy can exert differential effects on brachial and central pressures [13].

Arterial wall stiffness and reduced arterial compliance and dilation capacity are the first signs of cardiovascular diseases. Aortic pulse wave velocity (aPWV) is a measure of arterial stiffness and is reliably reproducible [14]. Data from prospective observational cohort studies indicate that aPWV relates to future cardiovascular risk even after accounting for other accepted cardiovascular risk factors such as peripheral BP [15]. Moreover, young healthy subjects with familial risk for hypertension showed increased arterial stiffness before any increase in BP $[11,16]$. In the PANIC-Study, poor cardio-respiratory fitness was independently associated with increased arterial stiffness and impaired arterial dilation capacity already among pre-pubertal children [5]. Augmentation index (AIx $=$ augmentation pressure/pulse pressure) represents pulse wave reflection and has been shown to be closely correlated to various cardiovascular risk scores [17]. Measures of arterial compliance are used to detect first signs of cardiovascular diseases even in children. However, the effects of a long term exercise intervention program on measures of arterial stiffness have not been evaluated in this young age group.

Therefore, the current study was designed to determine the influence of a long-term physical activity program on hemodynamic function and motor-skills in elementary school children who participated in the prevention-program "Fitness für Kids- Frühprävention im Kindergarten- und Grundschulalter" (Fitness for Kids - Early Prevention in Pre-school and Elementary School Children).

\section{Methods}

\subsection{Subjects and Study Design}

For the cluster-randomized controlled trial, students were recruited from a pool of participating "project schools" of the exercise program "Fitness für Kids". A primary school was randomly selected and two second grade classes were randomized either to intervention class (INT, $n=23$ ) or control class (CON, $n=22$ ). Forty-five pre-pubertal children (aged $6.6 \pm 0.7,29$ boys, 16 girls) were included in the study.

Apart from the school's principal and teachers, the participating students and their parents were informed about the course and the content of the study. Written parental consent was obtained prior to the study. The study was in agreement with the Helsinki Declaration on the use of human subjects for research and was submitted to and approved by the ethics committee of the Education Authority Berlin (Landesschulamt Berlin).

Prior to the intervention, baseline examinations were performed followed by a 9 months intervention period and a post-intervention examination, where all baseline measurements were repeated.

\subsection{Measurements}

Prior to the study, the subjects were familiarized with all experimental procedures and were subsequently tested on two different days. The first day, anthropometric measurements including body mass, height and waist circumference were registered to calculate body mass index (BMI), and waist to height 
ratio (WHtR). Children's motor performance was assessed using a motor skill test (Deutscher-Motorik-Test DMT 6-18 [18]). This test originally consists of 8 test items (20-meter sprint, side jumps, stand and reach, push-ups, sit-ups, dynamic balance, standing long jump, 6-minute endurance run) to evaluate the motor skills endurance, strength, speed, coordination and agility [18]. The test item dynamic balance could not be performed due to local reasons. For analyzing motor performance, a composite score was calculated by summing up the entire mean z-transformed item scores (tMP) [18]. Specially trained assistants in a station operation performed all tests in the school's gym, where all children followed the same station order.

On a separate day, hemodynamic parameters were determined non-invasively using Mobil-O-Graph ${ }^{\circledR}$ (24h PWA monitor, I.E.M., Stolberg GERMANY), a clinically validated device for hemodynamic measurements [19] with a novel transfer function-like algorithm, using brachial cuff-based waveform recordings. Peripheral and central BP, aPWV and AIx, a measure of pulse wave reflection, were assessed. To guarantee standardized conditions, the recommendations of the Clinical Applications of Arterial Stiffness, Task Force III [20] were followed. Subjects rested in a separate temperature controlled room $\left(23 \pm 1^{\circ} \mathrm{C}\right)$ for 10 minutes before two resting measurements were performed on each child on the right upper arm using custom fit arm cuffs. During measurements, the children had to extend their arms and place them on a customized arm-support, with the heart and the pressure cuff at the same level. The same investigator performed all BP measurements with the same device at the corresponding time of day pre- and post-intervention. The average of both measurements was used for analysis. To ensure that the children remained quiet during the measurement procedure, a short movie sequence ("March of the Penguins" Wild Bunch \& National Geographic Films) was presented.

\subsection{Intervention}

Throughout 9-months, both groups received the regular physical education class $(3 \times 45$ minutes/week $)$. Children of the INT participated in an additional exercise intervention consisting of a supervised physical activity program two times a week. Qualified and in intensive workshops specifically trained physical education teachers conducted the program. The content of the intervention was based on the movement concept of the prevention program "Fitness für Kids", which is characterized by moderate interval exercise activities. Short bouts of exercise, interspersed by rest periods, allowed relatively high intensity levels and corresponded to the child's general movement behavior. The standardized structure contained an initial worm-up followed by the main part consisting of different joyful games, activities, relays, coordination tasks, and a short cool-down thereafter. All contents were appropriate for children and could easily be performed in the school's gym irrespective of special equipment. The focus of the exercise sessions was a high degree of active movement time, which clearly stands out from the contents of general school physical education classes.

\subsection{Statistical Analyses}

All statistical analyses were performed using IBM SPSS Statistics 22.0 (SPSS, Chicago, IL, USA). Results are reported as means with one standard deviation $(S D)$ and confidence intervals. Paired Student's $t$-test was used to compare pre-intervention and post-intervention variables within the groups. Independent Student's $t$-tests assessed differences in mean change between INT and CON for all measured variables.). A $P$-value $<0.05$ was considered to indicate statistical significance.

\section{Results}

The characteristics of the exercise and control groups are in Table 1. INT displayed a lower height, weight, BMI and waist circumference at baseline 
compared with CON.

At baseline motor performance-scores were comparable in both groups (Table 2). This was true for hemodynamic measurements as well (Table 2). In both gender groups mean BP was above the 90th percentile, thus the sample can be classified "high-normal" [21]. After the intervention period, there were significant improvements in various test items of the motor skill test (20-meter sprint, side jumps, push-ups and sit-ups, tMP) in INT and CON. Concerning the stand and reach and 6-minute endurance run, only INT displayed considerable improvements. After the intervention period, INT demonstrated significantly higher scores in the side jumps $(P=0.03)$, stand and reach $(P=0.025)$, push-ups $(P=0.007)$, 6-minute endurance run $(P<$ $0.001)$ and tMP $(P<0.001)$ compared to CON (Table 2$)$.

Table 1 Subject's characteristics at baseline.

\begin{tabular}{lllll}
\hline Items & Total $(n=45)$ & INT $(n=23)$ & CON $(n=22)$ & $P$ values \\
\hline Boys/Girls $(n)$ & $29 / 16$ & $14 / 9$ & $15 / 7$ & 0.258 \\
Age $(\mathrm{yrs})$ & $6.6 \pm 0.7$ & $6.4 \pm 0.7$ & $6.7 \pm 0.7$ & 0.002 \\
Height $(\mathrm{cm})$ & $126.5 \pm 6.9$ & $123.0 \pm 7.0$ & $130.0 \pm 6.8$ & 0.002 \\
Weight $(\mathrm{kg})$ & $25.3 \pm 5.4$ & $22.4 \pm 3.9$ & $28.1 \pm 6.8$ & 0.026 \\
Body-Mass-Index $\left(\mathrm{kg} \cdot \mathrm{m}^{-2}\right)$ & $15.6 \pm 2.5$ & $14.7 \pm 1.8$ & $16.5 \pm 3.1$ & 0.016 \\
Waist circumference $(\mathrm{cm})$ & $57.9 \pm 5.8$ & $55.6 \pm 3.9$ & $60.2 \pm 7.6$ & 0.453 \\
Waist-to-height-ratio & $0.46 \pm 0.04$ & $0.45 \pm 0.03$ & $0.46 \pm 0.05$ & \\
\hline
\end{tabular}

Values are means $\pm 1 \mathrm{SD}$, unless stated otherwise.

Table 2 Motor performance test scores and hemodynamic measurements before (pre) and after (post) intervention.

\begin{tabular}{|c|c|c|c|c|c|c|c|c|}
\hline \multirow[t]{3}{*}{ Items } & \multicolumn{4}{|c|}{$\begin{array}{c}\text { INT } \\
(n=23) \\
\end{array}$} & \multicolumn{4}{|c|}{$\begin{array}{c}\text { CON } \\
(n=22)\end{array}$} \\
\hline & \multirow{2}{*}{$\frac{\text { pre }}{\mathrm{M} \pm \mathrm{SD}}$} & \multicolumn{3}{|l|}{ post } & \multirow{2}{*}{$\frac{\text { pre }}{\mathrm{M} \pm \mathrm{SD}}$} & \multicolumn{3}{|l|}{ post } \\
\hline & & $\mathrm{M} \pm \mathrm{SD}$ & $95 \%$ CI & $P$ value & & $\mathrm{M} \pm \mathrm{SD}$ & $95 \% \mathrm{CI}$ & $P$ value \\
\hline $\mathrm{SP}(\mathrm{sec})$ & $5.2 \pm 0.5$ & $4.7 \pm 0.3$ & $0.4 ; 0.7$ & $<0.001$ & $5.1 \pm 0.6$ & $4.7 \pm 0.4$ & $0.2 ; 0.6$ & $<0.001$ \\
\hline SJ (n jumps) & $23.3 \pm 5.7$ & $33.4 \pm 6.3 *$ & $-13.0 ;-7.2$ & $<0.001$ & $23.8 \pm 7.1$ & $28.6 \pm 8.2$ & $-7.3 ;-2.3$ & 0.001 \\
\hline $\mathrm{SR}(\mathrm{cm})$ & $0.5 \pm 6.2$ & $4.0 \pm 4.8^{*}$ & $-6.2 ;-0,9$ & 0.010 & $-1.4 \pm 5.0$ & $-1.0 \pm 5.6$ & $-1.3 ; 0.4$ & 0.329 \\
\hline PU (rep) & $11.0 \pm 5.0$ & $19.0 \pm 4.6 * *$ & $-8.9 ;-5.5$ & $<0.001$ & $12.8 \pm 3.8$ & $15.3 \pm 4.4$ & $-3.9 ;-1.1$ & 0.001 \\
\hline SU (rep) & $14.6 \pm 6.7$ & $19.9 \pm 5.7$ & $-6.8 ;-3.7$ & $<0.001$ & $15.0 \pm 5.8$ & $18.4 \pm 6.5$ & $-5.1 ;-1.6$ & 0.001 \\
\hline $\mathrm{LJ}(\mathrm{cm})$ & $109.0 \pm 21.4$ & $113.7 \pm 19.3$ & $-11.2 ; 1.9$ & 0.153 & $110.5 \pm 17.5$ & $113.7 \pm 15.2$ & $-7.8 ; 1.4$ & 0.160 \\
\hline $\mathrm{ER}(\mathrm{m})$ & $814.5 \pm 123.3$ & $916.7 \pm 114.6 \dagger$ & $-135.4 ;-69.0$ & $<0.001$ & $752.3 \pm 88.6$ & $753.7 \pm 117.2$ & $-54.4 ; 51.5$ & 0.955 \\
\hline tMP & $101.4 \pm 8.2$ & $108.3 \pm 5.4 \dagger$ & $-9.0 ;-5.0$ & $<0.001$ & $100.0 \pm 6.1$ & $102.0 \pm 5.8$ & $-3.6 ; 0.4$ & 0.019 \\
\hline $\mathrm{SBP}(\mathrm{mm} \mathrm{Hg})$ & $111.8 \pm 10.5$ & $110.0 \pm 9.2 *$ & $0.1 ; 3.7$ & 0.037 & $113.6 \pm 6.5$ & $115.5 \pm 8.5$ & $-4.8 ; 1.1$ & 0.196 \\
\hline $\mathrm{DBP}(\mathrm{mm} \mathrm{Hg})$ & $67.0 \pm 11.1$ & $64.7 \pm 8.4^{*}$ & $-0.4 ; 5.1$ & 0.091 & $68.3 \pm 5.8$ & $71.1 \pm 8.4$ & $-4.8 ;-0.7$ & 0.009 \\
\hline $\mathrm{cSBP}(\mathrm{mm} \mathrm{Hg})$ & $98.5 \pm 8.6$ & $96.8 \pm 7.3$ & $0.2 ; 3.2$ & 0.032 & $97.5 \pm 7.1$ & $97.9 \pm 7.9$ & $-2.6 ; 1.9$ & 0.744 \\
\hline $\mathrm{cDBP}(\mathrm{mm} \mathrm{Hg})$ & $69.5 \pm 11.7$ & $67.3 \pm 9.2 *$ & $-0.5 ; 5.0$ & 0.103 & $70.4 \pm 5.6$ & $72.6 \pm 8.0$ & $-3.9 ;-0.4$ & 0.019 \\
\hline $\operatorname{aPWV}\left(\mathrm{m} \cdot \mathrm{s}^{-1}\right)$ & $4.50 \pm 0.3$ & $4.44 \pm 0.3 *$ & $0.0 ; 0.1$ & 0.047 & $4.56 \pm 0.2$ & $4.64 \pm 0.3$ & $-0.2 ;-0.0$ & 0.187 \\
\hline AIx & $36.6 \pm 8.7$ & $37.2 \pm 9.5$ & $-4.9 ; 3.6$ & 0.759 & $31.6 \pm 8.8$ & $30.7 \pm 7.4^{*}$ & $-1.7 ; 3.3$ & 0.493 \\
\hline
\end{tabular}

Motor performance scores and hemodynamic measurements for intervention (INT) and control (CON) group before (pre) and after (post) intervention period. Values are means $(\mathrm{M}) \pm 1 \mathrm{SD}$.

Differences between INT and CON are displayed as $* P<0.05, * * P<0.01, \uparrow P<0.001$.

Abbreviations: $\mathrm{CI}=$ confidence interval; $\mathrm{SP}=20$-meter sprint; $\mathrm{SJ}=$ side jumps; $\mathrm{SR}=$ stand and reach; $\mathrm{PU}=$ push-ups; $\mathrm{SU}=$ sit-ups; $\mathrm{LJ}=$ standing long jump; $\mathrm{ER}=6$-minute endurance run; tMP = total motor performance $\mathrm{z}$-score; SBP = peripheral systolic blood pressure; $\mathrm{DBP}=$ peripheral diastolic blood pressure; $\mathrm{cSBP}=$ central systolic blood pressure; $\mathrm{cDBP}=$ central diastolic blood pressure; aPWV = aortic pulse wave velocity; AIx = Augmentation Index; rep = repetitions. 
When comparing the mean improvements between pre- and post-observation in both groups, there were significant differences in the side jumps $(P=0.007)$ push-ups $(P<0.001)$, 6-minute endurance run $(P=$ $0.001)$ and tMP $(P<0.001)$ favoring INT (Table 3, Fig. 1).

Table 3 Effects of intervention on motor performance test scores and hemodynamic measurements.

\begin{tabular}{|c|c|c|c|c|}
\hline \multirow[t]{2}{*}{ Items } & \multicolumn{2}{|c|}{ Change from baseline } & \multirow[b]{2}{*}{$95 \% \mathrm{CI}$} & \multirow[b]{2}{*}{$P$ value } \\
\hline & INT $(n=23)$ & $\mathrm{CON}(n=22)$ & & \\
\hline $\mathrm{SP}(\mathrm{sec})$ & $-0.5 \pm 0.3$ & $-0.4 \pm 0.4$ & $-0.4 ; 0.04$ & 0.105 \\
\hline SJ (n jumps) & $+10.1 \pm 6.9$ & $+4.8 \pm 5.6$ & $1.5 ; 9.0$ & 0.007 \\
\hline $\mathrm{SR}(\mathrm{cm})$ & $+3.5 \pm 6.2$ & $+0.4 \pm 1.9$ & $0.4 ; 5.8$ & 0.025 \\
\hline PU (rep) & $+7.2 \pm 4.0$ & $+2.5 \pm 3.2$ & $2.5 ; 6.8$ & $<0.001$ \\
\hline SU (rep) & $+5.2 \pm 3.6$ & $+3.4 \pm 3.9$ & $-0.3 ; 4.1$ & 0.092 \\
\hline $\mathrm{LJ}(\mathrm{cm})$ & $+4.7 \pm 15.4$ & $+3.2 \pm 10.4$ & $-6.5 ; 9.3$ & 0.715 \\
\hline $\mathrm{ER}(\mathrm{m})$ & $+102.2 \pm 78.7$ & $+1.5 \pm 119.4$ & $41.1 ; 160.4$ & 0.001 \\
\hline tMP & $+6.9 \pm 4.9$ & $+1.9 \pm 3.6$ & $2.4 ; 7.6$ & $<0.001$ \\
\hline $\mathrm{SBP}(\mathrm{mm} \mathrm{Hg})$ & $-1.9 \pm 4.1$ & $+1.9 \pm 6.6$ & $-7.1 ;-0.5$ & 0.026 \\
\hline DBP (mm Hg) & $-2.3 \pm 6.3$ & $+2.8 \pm 4.5$ & $-8,4 ;-1.8$ & 0.004 \\
\hline cSBP (mm Hg) & $-1.7 \pm 3.5$ & $+0.4 \pm 5.0$ & $-4.7 ; 0.6$ & 0.122 \\
\hline cDBP (mm Hg) & $-2.3 \pm 6.2$ & $+2.1 \pm 3.9$ & $-7.5 ;-1.2$ & 0.008 \\
\hline $\operatorname{aPWV}\left(\mathrm{m} \cdot \mathrm{s}^{-1}\right)$ & $-0.06 \pm 0.1$ & $+0.08 \pm 0.3$ & $-0.3 ;-0.008$ & 0.043 \\
\hline AIx & $+0.6 \pm 9.5$ & $-0.8 \pm 5.7$ & $-3.3 ; 6.3$ & 0.534 \\
\hline
\end{tabular}

Changes from baseline to post-intervention in motor performance test scores and hemodynamic measurements for intervention (INT) and control $(\mathrm{CON})$ group. Values are reported as means $(\mathrm{M}) \pm 1 \mathrm{SD}$. Abbreviations: $\mathrm{CI}=$ confidence interval; $\mathrm{SP}=20$-meter sprint; $\mathrm{SJ}$ = side jumps; $\mathrm{SR}=$ stand and reach $; \mathrm{PU}=$ push-ups; $\mathrm{SU}=$ sit-ups; $\mathrm{LJ}=$ standing long jump; $\mathrm{ER}=6$-minute endurance run; $\mathrm{tMP}=$ total motor performance $\mathrm{z}$-score; $\mathrm{SBP}=$ peripheral systolic blood pressure; $\mathrm{DBP}=$ peripheral diastolic blood pressure; $\mathrm{cSBP}=\mathrm{central}$ systolic blood pressure; $\mathrm{cDBP}=$ central diastolic blood pressure; aPWV = aortic pulse wave velocity; AIx = Augmentation Index; rep = repetitions.

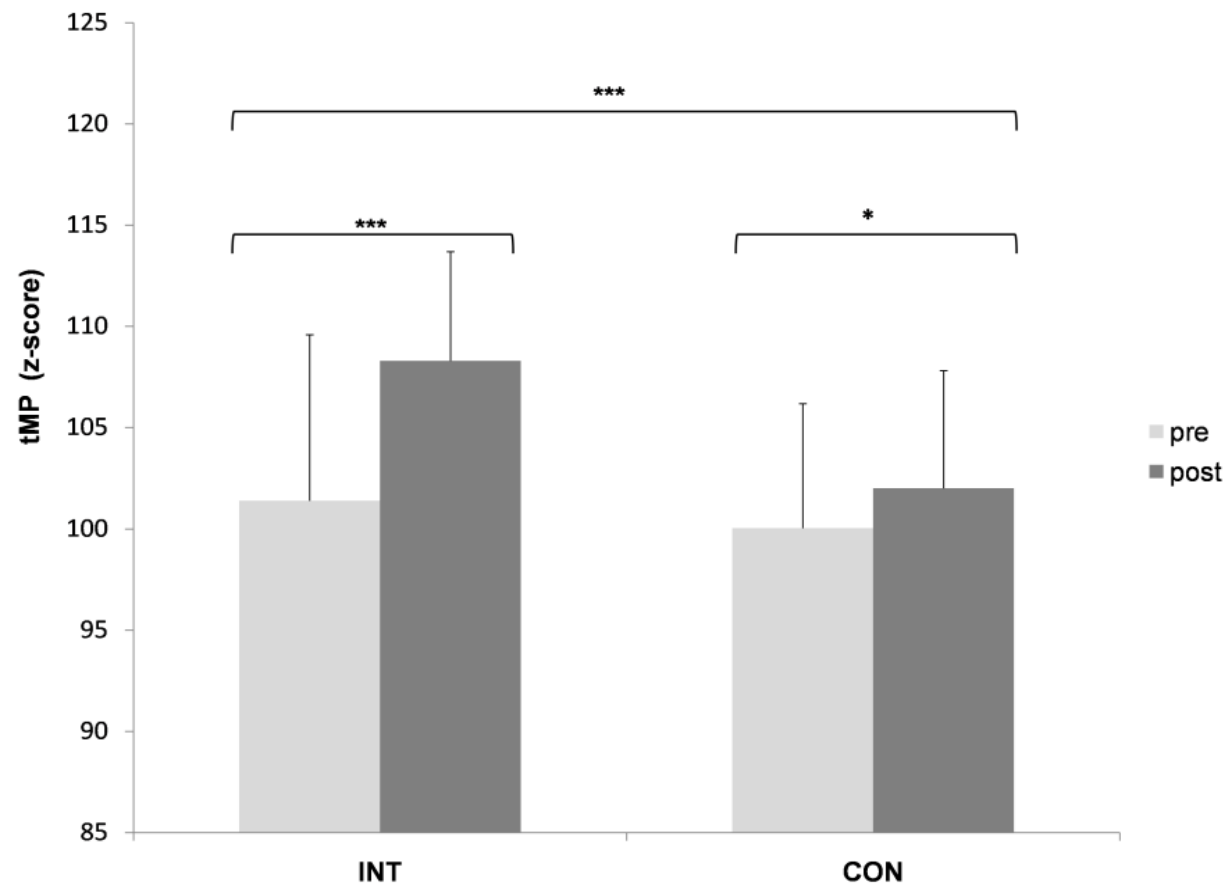

Fig. 1 Total motor performance Z-score (tMP) for intervention (INT) and control group (CON) before (pre) and following (post) intervention period. Values are means \pm 1 SD. $* P<0.05, * * * P<0.001$. 


\subsection{Hemodynamic Measurements}

Peripheral and central systolic pressures decreased significantly $(P=0.037 ; P=0.032)$ in INT. In contrast, CON both peripheral and central BP increased with a significant change in diastolic pressures $(P=0.009 ; P$ $=0.019)($ Table 2). Furthermore, aPWV was significantly $(P=0.047)$ lower in INT after intervention, whereas in CON aPWV was higher than before, thus resulting in a significant difference in the mean change between the two groups $(P=0.043)$ (Fig. 2). Both before and after intervention, AIx was higher in INT than in CON. There were no significant changes in AIx in both groups throughout the observation-period (Fig. 3, Table 3).

\section{Discussion}

The present study demonstrates beneficial effects of a 9 months exercise intervention on hemodynamic function and motor performance. The decrease in peripheral BP in INT and the difference between the two groups after 9 months exceeds the effects that have been found in adult study groups [22]. In a recently published study in adolescent schoolchildren, participants of the intervention group already displayed a significant decrease in systolic BP of five $\mathrm{mmHg}$ after 7 weeks of a high intensive interval training, whereas diastolic BP remained unchanged [23].

Elevated arterial pressure is the most treatable cause of premature death in developed countries. A family history of hypertension is a contributory risk factor, as is tachycardia, but neither is as strong a predictor as a youthful elevated pressure [24]. Due to data of the NHANES-Survey, BP increased substantially among children and adolescents in the United States [25] and increasing BP levels during childhood have been shown to strongly predict hypertension in young adulthood [26]. Independent of BMI, family history and socioeconomic factors, aerobic capacity in adolescence was an important factor associated with the long-term risk of hypertension in adulthood according to a recently published article [3]. Despite well-established benefits of regular physical activity on health and well-being, current levels within school aged youth are widely regarded as insufficient to meet

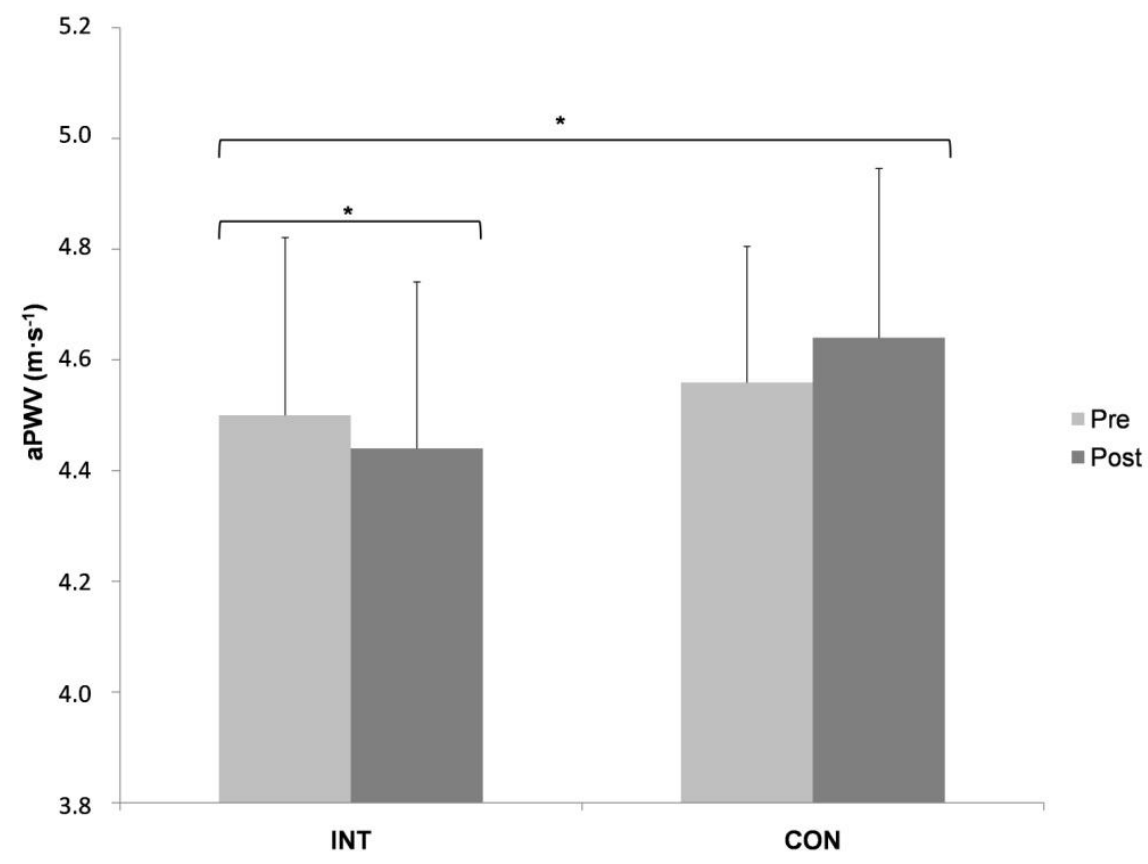

Fig. 2 Aortic pulse wave velocity (aPWV) for intervention (INT) and control group (CON) before (pre) and following (post) intervention period. Values are means \pm 1SD. $* P<0.05$. 


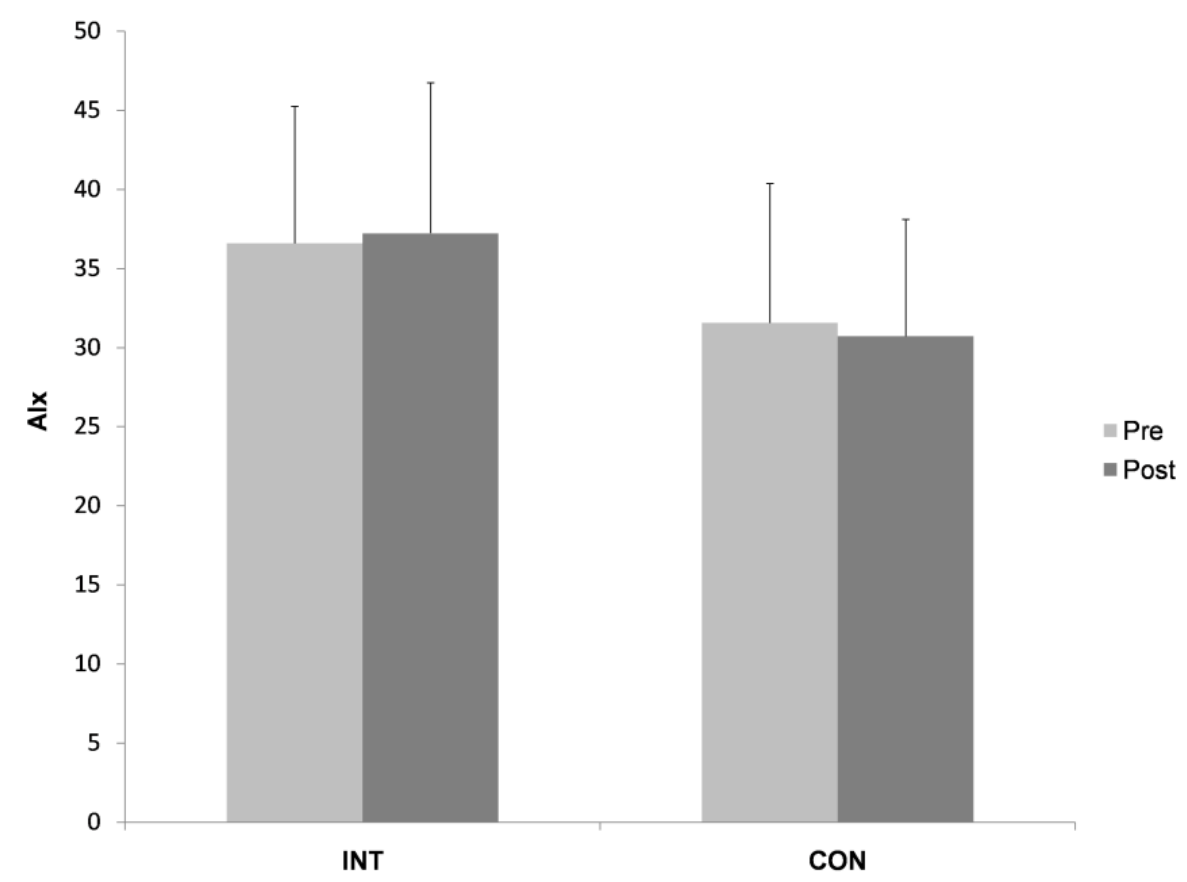

Fig. 3 Augmentation Index (AIx) for intervention (INT) and control group (CON) before (pre) and following (post) intervention period. Values are means \pm 1 SD.

recommendations [27].

According to the result of the current study, it can be concluded that a major potential benefit of regular physical activity is the prevention of the development of hypertension. Therefore, regular physical activity should be increased to improve cardiovascular risk profile and cardiopulmonary capacity and strengthen muscles and bones. The school environment, and in particular physical education classes, afford an ideal setting to practice health-promoting behaviors, where interventions can reach a large number of individuals from assorted socio-economic surroundings.

In the current study, systolic and diastolic central BPs decreased in INT whereas in CON both pressures were even higher after the observation period. In adults, central BP, the pressure in the aorta, has been shown to be more strongly related to preclinical organ damage, vascular disease, left ventricular hypertrophy and carotid atherosclerosis as markers of hypertensive end-organ damage and future cardiovascular events than the traditional upper arm BP $[12,13,28]$. The CAFE-study demonstrated better clinical outcome with comparable peripheral BP but with lower central BP due to medical therapy [29]. So far, to the best of our knowledge there are no data in children concerning central BP and its influence on cardiovascular outcome besides peripheral BP.

The same tendency was found in aPWV, which is widely measured as an indicator of arterial stiffness. Pulse wave depends on vessel size and the elastic properties of the aortic wall and predicts cardiovascular events and mortality in the general population beyond conventional risk factors [30]. Aortic stiffness increases with age especially in large and central arteries, and is accelerated by high BP [31]. The predictive value of aPWV is stronger in younger versus older subjects [15]. Therefore, the present results demonstrate that the expected age related increase in aPWV is not only compensated but even more, aPWV was reduced in INT after the intervention period. The difference in aPWV between INT and CON seen after the observation period was $0.2 \mathrm{~m} / \mathrm{sec}$. According to adult studies, this would translate into a $3 \%$ higher future cardiovascular risk in $\mathrm{CON}$.

On one side, the reduced aPWV in INT might be due to their lower BP, on the other side one can argue that 
this finding was due to an improved vascular function as well.

The Panic-Study showed that better physical fitness, physical activity and a low body fat percentage were associated with more flexible arterial walls already in primary school children and that only physical fitness was independently linked to arterial stiffness [5].

In healthy adults, increased arterial stiffness was superior to BP in predicting cognitive decline in all domains and in explaining the hypertension-executive function association [32]. Furthermore, previous studies suggest that aPWV attenuation is associated with improved survival [33].

Surprisingly and in contrast to the literature, adherence to the exercise program in the current study was not associated with a significant decrease in AIx, an index of arterial stiffness and wave reflection. It can be argued that probably the exercise intensity was not high enough to influence sufficiently the reduction in the expression of the elastin-degrading enzyme matrix metalloproteinase (MMP), since MMP plays a major role in the return of the reflected wave as one influential mechanism of arterial stiffness [34]. Furthermore, it has to be mentioned, that AIx was higher in INT than in CON, but the small difference was not statistically significant. This finding might be due the taller subjects in CON, since AIx, but not aPWV decreases with increasing height [35]. Another explanation might be the higher percentage of females in the INT-group, since females display higher stiffness than males during the pre-pubertal years [36]. Similarly, a study by Lurbe et al. [37] including children from 7 to 18 years reported a higher augmentation index in females compared to males.

It is widely believed that the performance of children and adolescents in physical fitness tests has declined over the past decades. A meta-analysis of Tomkinson et al. [38] confirmed a very rapid decline in physical performance over the last 20 years in children and adolescents aged 6-19 years. Early motor development and subsequently increased motor skill acquisition are predictors of regular physical activity during later childhood and adolescence [39].

The results of the current study show an improvement in tMP in INT, which serves as a measure of all physical test items. This variable was mainly influenced by the 6-minute endurance run and confirms an improvement in aerobic capacity due to the regular moderate interval training. It is in agreement with an earlier study in younger children, where nearly the same results came out after one year with three training sessions per week [40]. Better developed movement skills in early childhood are important contributors to the child's future physical and fitness level and can positively influence organized sports activities during later childhood and adolescence and future overall health [39].

Most participants who were physically active slacked off after the study's structured exercise program ended. Therefore, one should focused not only on motivation and to start as early as possible to adopt a healthy behavior but moreover, to integrate regular exercise into everybody's daily life. The present study draws attention to the substantive and independent value of physical activity on motor skills and, moreover, on various hemodynamic parameters demonstrating a beneficial effect for arterial health and therefore highlighting its significance as a tool for individual and population-based interventions.

One limitation of the study is the low number of subjects and physical fitness has not been evaluated before inclusion in the study. Furthermore, the level of participation in the regular physical education class in both groups and the level of adherence by the INT were not assessed. No other risk factors but body weight, BMI and WHtR have been examined in the present study. Therefore, the results do not provide information whether the beneficial effect of regular exercise derives from the influence of exercise itself or results from its effects on other cardiovascular risk factors, such as lipids and lipoproteins, who themselves are known to influence endothelial function. Before one can 
recommend the adoption of hemodynamic measurements in daily routine in such a young age group, randomized controlled trials are required to provide convincing evidence that this method has clinical value. Another limitation of this study is that given the extended period of the study it is possible that other confounding factors will have influenced the outcomes, which are not considered. Furthermore, the heterogeneity of the groups concerning anthropometric data might cause bias on the results. However, despite significant differences in BMI and WHtR, there were no significant differences in the hemodynamic parameters at baseline. This may lead to the assumption, that biometrical dimensions do not have such a strong influence on hemodynamic parameters in this young age and group differences could be neglected.

Overall, the results showed that higher levels of physical activity, even in early childhood were associated with better motor performance and hemodynamic parameters. Therefore, children should be encouraged to increase their participation in physical activity as early as possible and more policies promoting physical activity in this young population are needed to avoid unhealthy behaviors and reduce the burden of future cardiovascular diseases. Further research should access the potentials of regular exercise with higher intensities even in children, as been used in the present study. Probably similar favorable effects could be elicited through more intense interventions as already shown in adults. Apart from the high quality of the intervention in the present study, the interval exercise with the relatively high intensities may account for the more positive effects of this study then found elsewhere.

\section{Acknowledgments}

We would like to thank the participants and the teachers without whom this study would not have been possible.

This work has been funded by Takeda Pharma Company, Germany and I.E.M. Germany who provided the blood pressure measurement devices. Financial support was provided by the German Health Insurance Kaufmännische Krankenkasse Hannover, GERMANY due to subsidizing the preventive program "Fitness für Kids".

\section{References}

[1] Kearney, P. M., Whelton, M., Reynolds, K., Muntner, P., Whelton, P. K., and He, J. 2015. "Global Burden of Hypertension: Analysis of Worldwide Data." Lancet 365 (9455): 217-23.

[2] Huang, Y., Su, L., Cai, X., Mai, W., Wang, S., Hu, Y., Wu, Y., Tang, H., and Xu, D. 2014. "Association of All-Cause and Cardiovascular Mortality with Prehypertension: A Meta-Analysis." Am. Heart J. 167 (2): 160-8.

[3] Whelton, P. K., He, J., Appel, L. J., Cutler, J. A., Havas, S., Kotchen, T. A., Roccella, E. J., Stout, R., Vallbona, C., Winston, M. C., and Karimbakas, J.; National High Blood Pressure Education Program Coordinating Committee. 2002. "Primary Prevention of Hypertension: Clinical and Public Health Advisory from the National High Blood Pressure Education Program." JAMA 288 (15): 1882-8.

[4] Stary, H. C. 1989. "Evolution and Progression of Atherosclerotic Lesions in Coronary Arteries of Children and Young Adults." Arteriosclerosis 9 (1): 119-32.

[5] Veijalainen, A., Tompuri, T., Haapala, E. A.,Viitasalo, A., Lintu, N., Väistö, J., Laitinen, T., Lindi, V., and Lakka, T. A. 2016. "Associations of Cardiorespiratory Fitness, Physical Activity, and Adiposity with Arterial Stiffness in Children.” Scand. J. Med. Sci. Sports 26 (8): 943-50.

[6] Gray, L., Lee, I. M., Sesso, H. D., and Batty, G. D. 2011. "Blood Pressure in Early Adulthood, Hypertension in Middle Age, and Future Cardiovascular Disease Mortality: HAHS (Harvard Alumni Health Study).” J. Am. Coll. Cardiol. 58 (23): 2396-403.

[7] Koebnick, C., Black, M. H., Wu, J., Martinez, M. P., Smith, N., Kuizon, B. D., Jacobsen, S. J., and Reynolds, K. 2013. "The Prevalence of Primary Pediatric Prehypertension and Hypertension in a Real-World Managed Care System.” J. Clin. Hypertens. (Greenwich) 15 (11): 784-92.

[8] Timmons, B. W., Leblanc, A. G., Carson, V., Connor Gorber, S., Dillman, C., Janssen, I., Kho, M. E., Spence, J. C., Stearns, J. A., and Tremblay, M. S. 2012. "Systematic Review of Physical Activity and Health in the Early Years (Aged 0-4 Years).” Appl. Physiol. Nutr. Metab. 37 (4): 773-92.

[9] Shah, R. V., Murthy, V. L., Colangelo, L. A., Reis, J., Venkatesh, B. A., Sharma, R., Abbasi, S. A., Goff, D. C. Jr, Carr, J. J., Rana, J. S., Terry, J. G., Bouchard, C., 
Sarzynski, M. A., Eisman, A., Neilan, T., Das, S., Jerosch-Herold, M., Lewis, C. E., Carnethon, M., Lewis, G. D., and Lima, J. A. 2016. "Association of Fitness in Young Adulthood with Survival and Cardiovascular Risk: The Coronary Artery Risk Development in Young Adults (CARDIA) Study." JAMA Intern. Med. 176 (1): 87-95.

[10] Crump, C., Sundquist, J., Winkleby, M. A., and Sundquist, K. 2016. "Interactive Effects of Physical Fitness and Body Mass Index on the Risk of Hypertension." JAMA Intern. Med. 176 (2): 210-6.

[11] Kucerová, J., Filipovsky, J., Staessen, J. A., Cwynar, M., Wojciechowska, W., Stolarz, K., Kuznetsova, T., Gasowski, J., Dolejsova, M., Grodzicki, T., Kawecka-Jaszcz, K., and Fagard, R. 2006. "Arterial Characteristics in Normotensive Offspring of Parents with or without a History of Hypertension." Am. J. Hypertens. 19 (3): 264-9.

[12] Kollias, A., Lagou, S., Zeniodi, M. E., Boubouchairopoulou, N., and Stergiou, G. S. 2016. "Association of Central versus Brachial Blood Pressure with Target-Organ Damage: Systematic Review and Meta-Analysis." Hypertension 67 (1): 183-90.

[13] Mc Eniery, C. M., Cockcroft, J. R., Roman, M. J., Franklin, S. S., and Wilkinson, I. B. 2014. "Central Blood Pressure: Current Evidence and Clinical Importance." Eur. Heart J. 35 (26): 1719-25.

[14] Mitchell, G. F., Hwang, S. J., Vasan, R. S., Larson, M. G., Pencina, M. J., Hamburg, N. M., Vita, J. A., Levy, D., and Benjamin, E. J. 2010. "Arterial Stiffness and Cardiovascular Events: The Framingham Heart Study." Circulation 121 (4): 505-11.

[15] Ben-Shlomo, Y., Spears, M., Boustred, C., May, M., Anderson, S. G., Benjamin, E. J., Boutouyrie, P., Cameron, J., Chen, C. H., Cruickshank, J. K., Hwang, S. J., Lakatta, E. G., Laurent, S., Maldonado, J., Mitchell, G. F., Najjar, S. S., Newman, A. B., Ohishi, M., Pannier, B., Pereira, T., Vasan, R. S., Shokawa, T., Sutton-Tyrell, K., Verbeke, F., Wang, K. L., Webb, D. J., Willum-Hansen, T., Zoungas, S., McEniery, C. M., Cockcroft, J. R., and Wilkinson, I. B. 2014. "Aortic Pulse Wave Velocity Improves Cardiovascular Event Prediction: An Individual Participant Meta-Analysis of Prospective Observational Data from 17,635 Subjects." J. Am. Coll. Cardiol. 63 (7): 636-46.

[16] Ciolac, E. G., Bocchi, E. A., Bortolotto, L. A., Carvalho, V. O., Greve, J. M., and Guimarãs, G. V. 2010. "Haemodynamic, Metabolic, and Neuro-humoralabormalities in Young Normotensive Women at High Familial Risk for Hypertension." J. Hum. Hypertens. 24 (12): 814-22.

[17] Nürnberger, J., Keflioglu-Scheiber, A., Opazo Saez, A. M.,
Wenzel, R. R., Philipp, T., and Schäfers, R. F. 2002. "Augmentation Indexis Associated with Cardiovascular Risk.” J. Hypertens. 20 (12): 2407-14.

[18] Bös, K., Schlenker, L., Büsch, D. D., Lämmle, L., Müller, H., Oberger, J., Seidel, I., and Tittlbach, S. 2009. German Motor Skill Test 6-18 [in German]. Hamburg: Czwalina.

[19] Franssen, P. M., and Imholz, B. P. 2015. "Evaluation of the Mobil-O-Graph New Generation ABPM Device Using the ESH Criteria." Blood Press Monit. 15 (4): 229-31.

[20] Van Bortel, L. M., Duprez, D., Starmans-Kool, M. J., Safar, M. E., Giannattasio, C., Cockcroft, J., Kaiser, D. R., and Thuillez, C. 2002. "Clinical Application of Arterial Stiffnes, Task Force III: Recommendations for User Procedures." American Journal of Hypertension 15 (5): 445-52.

[21] Lurbe, E., Cifkova, R., Cruickshank, J. K., Dillon, M. J., Ferreira, I., Invitti, C., Kuznetsova, T., Laurent, S., Mancia, G., Morales-Olivas, F., Rascher, W., Redon, J., Schaefer, F., Seeman, T., Stergiou, G., Wühl, E., and Zanchetti, A. 2009. "Management of High Blood Pressure in Children and Adolescents: Recommendations of the European Society of Hypertension." Journal of Hypertension 27 (9): 1719-42.

[22] Whelton, S. P., Chin, A., Xin, X., and He, J. 2002. "Effect of Aerobic Exercise on Blood Pressure: A Meta-Analysis of Randomized Controlled Trials." Ann. Intern. Med. 136 (7): 493-503.

[23] Buchan, D. S., Ollis, S., Young, J. D., Cooper, S. M., Shield, J. P. H., and Baker, J. S. 2013. "High Intensity Interval Running Enhances Measures of Physical Fitness but not Metabolic Measures of Cardiovascular Disease Risk in Healthy Adolescents." BMC Public Health 13: 498.

[24] Jamerson, K., and Julius, S. 1991. "Predictors of Blood Pressure and Hypertension. General Principles." Am. J. Hypertens. 4 (11): 598S-602S.

[25] Muntner, P., He, J., Cutler, J. A., Wildman, R. P., and Whelton, P. K. 2004. "Trends in Blood Pressure Among Children and Adolescents." JAMA 291 (17): 2107-13.

[26] Klumbiene, J., Sileikiene, L., Milasauskiene, Z., Zaborskis, A., and Shatchkute, A. 2000. "The Relationship of Childhood to Adult Blood Pressure: Longitudinal Study of Juvenile Hypertension in Lithuania." J. Hypertens. 18 (5): 531-38.

[27] Ekelund, U., Tomkinson, G., and Armstrong, N. 2011. "What Proportion of Youth Are Physically Active? Measurement Issues, Levels and Recent Time Trends." Br. J. Sports Med. 45 (11): 859-65.

[28] Shimizu, M., Hoshide, S., Ishikawa, J., Yano, Y., Eguchi, K., and Kario, K. 2015. "Correlation of Central Blood Pressure to Hypertensive Target Organ Damages During Antihypertensive Treatment: The J-TOP Study." Am. J. 
Hypertens. 28 (8): 980-6.

[29] Williams, B., Lacy, P. S., Thom, S. M., Cruickshank, K., Stanton, A., Collier, D., Hughes, A. D., Thurston, H., and O'Rourke, M.; CAFE Investigators; Anglo-Scandinavian Cardiac Outcomes Trial Investigators; CAFE Steering Committee and Writing Committee. 2006. "Differential Impact of Blood Pressure Lowering Drugs on Central Aortic Pressure and Clinical Outcomes: Principal Results of the Conduit Artery Function Evaluation (CAFE) Study." Circulation 113 (9): 1213-25.

[30] Willum-Hansen, T., Staessen, J. A., Torp-Pedersen, C., Rasmussen, S., Thijs, L., Ibsen, H., and Jeppesen, J. 2006. "Prognostic Value of Aortic Pulse Wave Velocity as an Index of Arterial Stiffness in the General Population.” Circulation 113 (5): 664-70.

[31] Benetos, A., Adamopoulos, C., Bureau, J. M., Temmar, M., Labat, C., Bean, K., Thomas, F., Pannier, B., Asmar, R., Zureik, M., Safar, M., and Guize, L. 2002. "Determinants of Accelerated Progression of Arterial Stiffness in Normotensive Subjects and in Treated Hypertensive Subjects over a 6-Year Period." Circulation 105 (10): 1202-7.

[32] Hajjar, I., Goldstein, F. C., Martin, G. S., and Quyyumi, A. A. 2016. "Roles of Arterial Stiffness and Blood Pressure in Hypertension-Associated Cognitive Decline in Healthy Adults." Hypertension 67 (1): 171-5.

[33] Guerin, A. P., Blacher, J., Pannier, B., Marchais, S. J., Safar, M. E., and London, G. M. 2001. "Impact of Aortic Stiffness Attenuation on Survival of Patients in End-Stage Renal Failure." Circulation 103 (7): 987-92.

[34] Essteban-Cornejo, I., Hallal, P. C., Mielke, G. I., Menezes,
A. M., Gonçalves, H., Wehrmeister, F., Ekelund, U., and Rombaldi, A. J. 2015. "Physical Activity Throughout Adolescence and Cognitive Performance at 18 Years of Age." Med. Sci. Sports Exerc. 47 (12): 2552-7.

[35] Cameron, J. D., McGrath, B. P., and Dart, A. M. 1998. "Use of Radial Artery Applanation Tonometry and a Generalized Transfer Function to Determine Aortic Pressure Augmentation in Subjects with Treated Hypertension.” J. Am. Coll. Cardiol. 32 (5): 1214-20.

[36] Rossi, P., Frances, Y., Kingwell, B. A., and Ahimastos, A. A. 2011. "Gender Differences in Artery Wall Biomechanical Properties Throughout Life." J. Hypertens. 29 (6): 1023-33.

[37] Lurbe, E., Torro, M. I., Carvajal, E., Alvarez, V., and Redon, J. 2003. "Birth Weight Impacts on Wave Reflections in Children and Adolescents." Hypertension 41 (3 Pt 2): 646-50.

[38] Tomkinson, G. R., Léger, L. A., Olds, T. S., and Cazorla, G. 2003. "Secular Trends in the Performance of Children and Adolescents (1980-2000): An Analysis of 55 Studies of the 20m Shuttle Run Test in 11 Countries." Sports Med. 33 (4): 285-300.

[39] Barnett, L. M., van Beurden, E., Morgan, P. J., Brooks, L. O., and Beard, J. R. 2009. "Childhood Motor Skill Proficiency as a Predictor of Adolescent Physical Activity." J. Adolesc. Health 44 (3): 252-9.

[40] Ketelhut, K., Mohasseb, I., and Ketelhut, R. G. 2010. "Influences of a Regular Exercise Program on Blood Pressure and Blood Pressure Development at Rest and During Exercise and Motor Performance in Children." Schweiz Z Sportmed Sporttraum 58 (4): 115-9. 